Material and Method A retrospective statistic study of ADI in children aged $0-16$ years, admitted to $2^{\text {nd }}$ Pediatric Clinic, Emergency County Hospital Craiova, over a period of 6 years (01.01.2006-31.12.2011).

Results Of the total number of 14427 admitted children, aged 0-16 years, 645 presented acute intoxications with various etiologies, among which 252 had ADI; intoxication type: accidental in $154(61.1 \%)$, voluntary in $98(38.9 \%)$.

Accidental ADI: sex $M / F=54 / 100$, social environment $\mathrm{U} / \mathrm{R}=93 / 61$, age group (years): $0-1 / 1-3 / 3-6 / 6-10=27 / 68 / 33 / 26$.

Voluntary $\mathrm{ADI}$ : $\mathrm{M} / \mathrm{F}=23 / 75, \mathrm{U} / \mathrm{R}=59 / 39$, age (years): 6-10/ $10-14 / 14-16=7 / 37 / 54$

Etiologic spectrum in the studied group: AINS/antialgics in 39 (15.5\%), methoclopramid 29 (11.5\%), anti-epileptics 24 (9.5\%), tranquilizers/sedatives 15 (5.9\%), neuroleptics 9 (3.6\%), parasympathicolitics 8 (3.2\%), antibiotics/antiparasitary 17 (6.7\%), drugs with cardio-vascular effect 8 (3.2\%), drug combinations 41 $(16.3 \%)$, other drugs $12(4.8 \%)$, unknown 50 (19.8\%). Clinical forms: mild in 127 (50.4\%), moderate 101 (40.1\%) and severe 24 (9.5\%). No deaths were registered with ADI.

Average period of hospitalization: accidental ADI $3.33 \pm 2.54$ (1-9), voluntary ADI 3.28 \pm 1.24 (1-6) days.

Conclusions $\mathrm{ADI}$ represented $39.1 \%$ of the total number of acute intoxications; $61.1 \%$ of $\mathrm{ADI}$ were accidental. Most $\mathrm{ADI}$ were caused by AINS, methoclopramid, anti-epileptics. ADI prevailed in females, in urban children, both in voluntary and accidental ADI. The clinical forms were mostly mild.

\section{RELATIONSHIP BETWEEN MYOCARDIAL INJURY, OXIDATIVE STRESS MECHANISM AND SEPSIS/SEPTIC SHOCK IN INFANTS SUBMITTED TO SURGERY FOR CONGENITAL HEART DISEASE}

doi:10.1136/archdischild-2012-302724.1654

'MS Oliveira, 'EM Floriano, ${ }^{2} \mathrm{PH}$ Manso, ${ }^{3} \mathrm{RN}$ Sgabieri, ${ }^{4} \mathrm{JG}$ Klamt, ${ }^{5} \mathrm{EZ}$ Martinez, ${ }^{3} \mathrm{WVA}$ Vicente, 'SG Ramos. 'Department of Pathology; ${ }^{2}$ Department of Pediatrics; ${ }^{3}$ Department of Surgery and Anatomy; ${ }^{4}$ Department of Biomechanics, Medicine and Locomotive Apparatus Rehabilitation; ${ }^{5}$ Department of Statistics, Faculty of Medicine of Ribeirão Preto, University of São Paulo, Ribeirão Preto, Brazil

Background and Aims A progressive ventricular dysfunction caused by ischemic myocardial injuries remains one of the leading causes of death during the postoperative course in congenital heart disease (CHD). The aim of this study was to investigate the role of oxidative stress in these myocardial injuries.

Methods Myocardial injuries and oxidative stress mechanisms were assessed by histopathology and immunohistochemistry and quantified by morphometrical analyses.

Results Myocardial injury was observed in pediatric patients submitted to surgery for CHD with cardiopulmonary bypass, followed by lethal exit. Oxidative stress mechanisms were directly related to these particular types of myocardial injuries. Importantly, 4- hydroxinonenal (4-HNE), a marker of lipid peroxidation, is strongly expressed, especially in irreversible myocardial lesions. Although morphologically similar, myocardial injuries observed in patients who evolved with sepsis in the peri-operatory period exhibited a completely different set of oxidative stress mechanisms. Increased concentrations of nitrotyrosine protein adducts were observed in these patients, suggesting that peroxynitrite-mediated protein nitration may be the predominant oxidative stress mechanism found in these situations.

Conclusions The underlying mechanisms of these lesions seem to be related to the development of ischemia or ischemia/reperfusion followed by oxidative stress mechanisms that vary depending on whether sepsis was present. While the exact mechanism is not fully understood, it has been suggested that endogenous catecholamine release could have a role in this process.

\section{LEVOSIMENDAN AND MILRINONE: A SAFE COMBINATION?}

doi:10.1136/archdischild-2012-302724.1655

'A Morales Martínez, ${ }^{2} \mathrm{P}$ García Soler, ${ }^{1} \mathrm{C}$ Yun Castilla, 'L Romero Moreno, 'JM Camacho Alonso. 'Pediatric Intensive Care Unit, Hospital Regional Universitario Materno Infantil Carlos Haya; ${ }^{2}$ Hospital Materno Infantil Carlos Haya, Málaga, Spain

Background/Aims Levosimendan is an inotropic and vasodilator drug. Most protocols suggest avoiding other vasodilators, inodilators after its introduction. The technical data recommends not using with other vasodilators. We report our experience of concomitant use of levosimendan and milrinone in a series of patients.

Methods Observational study. Review of medical records of concomitant use of levosimendan (24 hours infusion without loading dose) and milrinone, from June 2009 to December 2011. Multivariant analysis of epidemiologic data, pathology, type of heart failure, indication of the drug and side effects.

Results 81 cases received levosimendan. Among them,64(79\%) received simultaneously milrinone. Mean age 14.8 months (5 days-112 months) $57.8 \%$ males.87.5\% were postoperative cardiac surgical patients (41\% tetralogy of Fallot). Right ventricular failure was the most common indication (56.9\%) followed by left ventricular failure (29.2\%) and biventricular failure (12.3\%).Diastolic dysfunction was reported in $49.2 \%$ of our patients, $46.1 \%$ systolic dysfunction and $4.6 \%$ both.In $31 \%$ of cases bothdrugswereinitiated simultaneously(operating theatre).Milrinone was the first drug in $41.5 \%$ cases. The average dose of milrinone was $0.8 \mathrm{mcg} / \mathrm{kg} / \mathrm{min}$. 19/63 cases (30\%) suffered from hypotension. In 7 cases (11\% of total) milrinone was suspended (without association to type of pathology or dysfunction). In the other 12 patients, infusion of milrinone was decreased, but not suspended. Among the cases of right ventricular failure, hypotension appeared in $32.4 \%$,in $10.5 \%$ with left ventricular failure and in $55.5 \%$ with biventricular. There were no complications associated.

Conclusions In our series, administration of levosimendan and milrinone was safe. The appearance of hypotension was controlled and was not associated with other complications. Patients at increased risk of hypotension were those with biventricular dysfunction. Larger prospective studies are necessary to test the safety of this combination.

\section{BIOMARKERS FOR SEPSIS, MULTIPLE ORGAN DYSFUNCTION SYNDROME (MODS) AND MORTALITY AFTER OPEN HEART SURGERY IN CHILDREN}

doi:10.1136/archdischild-2012-302724.1656

${ }^{1} \mathrm{~T}$ da Rocha, ${ }^{2} \mathrm{~L}$ Mulle, ${ }^{2} \mathrm{C}$ Ricachinevsky, ${ }^{2 \mathrm{~A}}$ Botta, ${ }^{1} \mathrm{P}$ Carvalho. ${ }^{1}$ Uti Pediatrica Hospital Santo Antonio, Universidade Federal do Rio Grande do Sul; 2Uti Pediatrica Hospital Santo Antonio, Santa Casa de Misericórdia de Porto Alegre, Porto Alegre, Brazil

Background and Aims Biomarkers can help to predict risk of unfavorable outcomes after open heart surgery in children.

Methods We performed a retrospective cohort of 121 children after open heart surgery. We analyzed the serum blood lactate, base excess (BE), blood glucose, central venous oxygen saturation (SATvc), troponin I, C-reactive protein (CRP), and leukocyte counts in different postoperative days (POd).

Results There were $7.4 \%$ deaths, $27.3 \%$ of sepsis and $60.3 \%$ of MODS. For death, showed better power PO1d and PO2d lactate $(\mathrm{OR}=24.1$ [CI 4-112]) and (OR = 9.7 [CI 1.2 to 85.7]); PO1d EB $(\mathrm{OD}=30.6$ [CI 2.6 to 351]); PO1d total leukocytes $(\mathrm{OD}=5.8$ (1.2 to 29.8]) For sepsis, showed better power: PO6h glucose $(\mathrm{OD}=2.4$ [1.03 to 5.7]); POI and PO3d SATvc (OD $=2.4$ (1.09 to 5.8]) and $(\mathrm{OD}=256$ [2.2-298]); PO6h troponin I (OD = 2.8 [1.1 to 6.8]) and PO1d bastonades (OD $=6.5$ [1.4 to 29.6 ]). For MODS, showed better power: $\mathrm{PO} 6 \mathrm{~h}$ and PO1d SATvc (OD $=12.2$ [2.6 to 55.7]) $(\mathrm{OD}=2.87$ [1.1 to 7.4$])$ and, $\mathrm{POI} / \mathrm{PO} 6 \mathrm{~h} / \mathrm{PO} 1 \mathrm{~d}$ troponin- $\mathrm{I}(\mathrm{OD}=3.2$ [1.6 to 8.0]); POI/PO6h CPR (OD = 3.7 [1.3 to 10.8]). 